\title{
University Students' Reasoning on Physical Information Encoded in Quantum State at a Point in Time
}

\author{
Marisa Michelini and Giacomo Zuccarini
}

Department of Chemistry, Physics and Environment, University of Udine, 33100 Udine, Italy

\begin{abstract}
Learning quantum mechanics entails adopting a new reference frame for the physical interpretation of the world. The quantum perspective is intrinsically connected with math, which becomes a sort of referent for physical meaning, requiring the employment of new formal structures and a new interpretation of familiar ones. Research evidences that students have difficulty both with concepts and with the use of formalism in qualitative tasks. We administered a 15-item questionnaire focused on incompatibility of observables and related formal structures to 40 physics students of three Italian universities. Semi-structured interviews were scheduled on a subset of students. Results concerning translation processes between math and physical meaning show that most students only look at the square modulus in order to reason on physical information encoded in quantum state, thus neglecting phase relations and their connection with incompatibility.
\end{abstract}

Keywords: quantum mechanics, quantum state, incompatible observables, physics education, student understanding PACS: 01.40.Fk, 03.65.-w, 03.65.Ca

\section{INTRODUCTION}

Building mental models of quantum concepts and of their interconnection is a difficult task even for upper division students. The interpretation of the physical behavior of micro-systems requires the adoption of new concepts such as incompatibility of observables, whose construction is non-intuitive, and implies the redefinition of basic notions such as the state of a system.

In turn, the structure of these new features is encoded in a highly mathematical formalism, which requires as well making physical meaning of new entities (e.g. the operator structure of observables) and the reinterpretation of familiar ones (e.g. vector superposition).

Students face therefore multiple layers of complexity: purely conceptual, technical, and structural ones, with the latter referring to the translation processes between mathematics and physical meaning [1].

Incompatibility is at the core of this complexity. From a conceptual point of view, it is a prerequisite to identify if eigenstates of a given observable are stationary or not and therefore their time evolution in the absence of measurement, as well as to assess the gain and loss of system properties in the measurement process. Consequently, a solid understanding of quantum behavior requires building an understanding of incompatibility according to the different roles it plays in quantum processes. In addition, from a structural point of view incompatibility is behind the adoption of the non-commutative algebra of operators, and heavily influences the way in which physical information is encoded in quantum state formalism.
Research evidences that students have difficulty mastering concepts and applying the formalism to answer qualitative questions [2]. More specifically, different studies elicited the importance of incompatibility in learning difficulties with some aspects of quantum behavior and with the role of formal entities. For instance, students resort to classical ideas such as energy conservation to describe the effects of quantum measurement, neglecting incompatibility [3]. They state that eigenstates of incompatible observables, such as energy and position, are coincident [4]. In dealing with time evolution, they ascribe stationarity to eigenstates of observables not commuting with energy and struggle to identify observables commuting with energy as constants of motion [5].

Nevertheless, even among basic elements of quantum mechanics (QM), some aspects of incompatibility have been barely touched by research. One case is represented by the role of phase relations in quantum state at a point in time. Every pure state can be written as linear combination of eigenstates of a complete set of compatible observables. In this perspective, the square modulus of the coefficients contains all information on these observables, while phase relations complete information on observables not commuting with at least an element of the set.

In order to explore student understanding of incompatibility on the conceptual and on the structural level, we conducted a research in different Italian universities. Here we report results concerning physical information encoded in quantum state formalism (role of square modulus, phase relations, and superposition), according to the following research questions (RQ): 
RQ1: how do students relate patterns of experimental data with the coefficients of $|\psi\rangle$ in the context of spin $1 / 2$ particles?

RQ2: how do they relate an initial superposition state with the eigenstates of the measured observable?

RQ3: how do they predict measurement outcomes of an observable not commuting with that on which $|\psi\rangle$ is expanded?

\section{INSTRUMENTS AND METHODS}

As this research is part of a project aimed at the construction of teaching/learning proposals devised to overcome student difficulties, we adopted the Model of Educational Reconstruction (MER) as theoretical framework [6]. According to MER, an essential step in the development of a teaching/learning sequence is clarification of science content, as well as research on student learning. Therefore, this project started with a first analysis of theoretical content focused on quantum state and of educational literature on student difficulties. On this basis, research instruments were developed by means of two calibration stages conducted with case study methods $[7,8]$.

Their results led us to a refinement of focus, i.e. to identify incompatibility of observables as a crucial aspect to be explored at different levels and from different perspectives. Subsequently, a rubric was elaborated on the topic, including both its conceptual role in measurement and in time evolution, and the structural role of formal entities connected with it.

The rubric was used to build a 15-item questionnaire exploring the above mentioned issues both in global terms, and in the application context of specific problems. Semi-structured interviews were scheduled on each questionnaire item

We administered the test to 40 physics student volunteers from three Italian universities (see Table I):

TABLE I. Participants per type and institution

\begin{tabular}{c|c|c}
\hline University & Type of students & Number \\
\hline $\begin{array}{c}\text { University of } \\
\text { Cagliari }\end{array}$ & $\begin{array}{c}3^{\text {rd }} \text { year } \\
\text { undergraduate }\end{array}$ & 10 \\
\hline $\begin{array}{c}\text { Roma Tre } \\
\text { University }\end{array}$ & $\begin{array}{c}3^{\text {rd }} \text { year } \\
\text { undergraduate }\end{array}$ & 10 \\
\hline $\begin{array}{c}\text { University of } \\
\text { Turin }\end{array}$ & $\begin{array}{c}3^{\text {rd }} \text { year } \\
\text { undergraduate }\end{array}$ & 12 \\
\cline { 2 - 3 } & graduate & 8 \\
\hline
\end{tabular}

Thirty two students had just completed a standard upper division QM course, including the discussion of spin, while the remaining eight were graduate students who already attended at least one advanced QM course. We interviewed a subset of six undergraduates from the above mentioned universities and two graduates.
Written test and interviews were analyzed according to qualitative research methods. Typical sentences and a-priori categories were built by identification of crucial conceptual contents and literature analysis on learning difficulties in QM. Categories were revised on the base of conceptual elements introduced by student answers. Emerging element clusters and coherence elements in student reasoning were identified.

As previous research indicates that most students struggle with similar concepts, regardless of instructor, textbook, or institution [9], the answers of undergraduate students from different universities were analyzed as a whole data set.

Each RQ is addressed by a specific item labeled with the same number and discussed in the next section.

\section{RESULTS}

Q1: A beam of silver atoms (spin 1/2) is identically prepared by an atomic beam source in an unknown spin state, which can be written as follows: $|\psi\rangle=\alpha \mid z+>$ $+\beta \mid z->$. The beam is sent through a Stern-Gerlach device measuring the spin component in the $\mathrm{z}$ direction (Sz). On the screen we observe two spots of equal intensity.

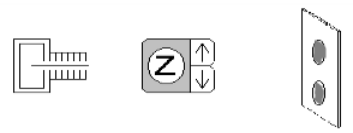

What do we learn about the coefficients $\alpha$ and $\beta$ of the state vector $\mid \psi>$ ? Describe your reasoning

FIGURE 1. Item Q1's text.

Two different kinds of answers were considered correct. The first - more complete - includes considerations on phase relations between $\alpha$ and $\beta$, such as "by choosing $\alpha$ real and positive, we get $\alpha=1 / \sqrt{2}$, while $\beta$ is defined up to a phase: $\beta=e^{i \varphi} 1 / \sqrt{2}$ ', and was given by six undergraduates. The second kind of answer, focusing only on the value of the square moduli, e.g. " $|\alpha|^{2}=|\beta|^{2}=1 / 2$ ", was given by one undergraduate and four graduates.

21/32 undergraduates and 3/8 graduates claimed that both coefficients are real positive numbers, e.g. " $\alpha=\beta=1 / \sqrt{2}$ ". Some of them reported at first the correct relation between the square moduli, and justified their conclusion as a result of a free choice on coefficients: "I chose $\alpha, \beta \in \mathfrak{R}^{+}$, so $\alpha=1 / \sqrt{2}=\beta$ ", but most described it as a natural consequence of experimental outcomes: "probability is the same, therefore coefficients are equal". These results are not surprising if we consider that even students giving correct answers underlined the physical irrelevance of phase relations: "the two coefficients differ only by a phase factor that is not physically interesting". 
Only two students didn't answer this item. The remaining five students didn't identify any relation between coefficients, trying instead to reconstruct the phenomenology of the experiment.

An important conceptual aspect of student answers is tied to the distinction between quantum measurement and property reconstruction. In fact, while in classical physics an ideal measuring device passively records a property of the system, quantum measurement is mostly an active process, where we come to know the property acquired by the system in the interaction with the device. Reconstructing the property (or properties) possessed by the system before the measurement is a different procedure in QM, closely tied to the empirical reconstruction of the initial state of the system.

Students ascribing real and positive value to coefficients at least implicitly mix up measurement and state reconstruction. Asked in the interview about state reconstruction, one of these students said: "Since we determined the coefficients, the state is fixed. [...] Here I chose real and positive coefficients, so theoretically I have no problem with phase." Three other students provided an explicit expression for the initial state vector: $|\psi\rangle=1 / \sqrt{2}(|z+>+| z->)$.

Q2: Consider the following statement referring to the experimental situation described in item Q1: "Before the Stern-Gerlach device, the atoms prepared by the source in the state $|\psi\rangle$ are in a Sz eigenstate". Express an opinion on the statement, explaining your reasoning.

FIGURE 2. Item Q2's text.

Acquiring a solid understanding of the concept of eigenstate as output state of a measurement is a crucial achievement for students, as it represents a fundamental junction between quantum state and the measurement process and - at a structural level - between the vector structure of the state and the operator structure of observables. Q2 discusses the connection between the concepts of superposition and eigenstate: if $|\psi\rangle$ is a superposition of eigenstates of $\mathrm{Sz}$ related to different eigenvalues, then it is not an eigenstate of Sz. While this is an elementary aspect of the eigenstate concept, 8/32 undergraduates left the answer blank.

Half of the students answered Q2 correctly. Among them there was an equal proportion of undergraduate (16/32) and graduate students (4/8). Some answers were based on a physical reasoning, e.g. "surely not [an eigenstate], otherwise we'd have observed only one spot on the screen." or "otherwise we'd always obtain the same result", others on a mathematical one: "they are in a superposition of eigenstates, therefore not in an eigenstate". Three answers evidenced a retrocausal thinking, i.e. the idea that state collapse already takes place in the Stern-Gerlach device, and not on the screen ("The two beams are in a well defined spin state, one in $|z+\rangle$ and the other in $\mid z->$ '). Anyway, these answers were considered correct with relation to the eigenstate concept.

Seven undergraduates and one graduate evidenced a passive conception of measurement, interpreting $|\psi\rangle$ as a statistical mixture of states. Both students agreeing with Q2 statement, and students rejecting it display this idea. It just depends whether they look at a single atom (e.g. "each atom is in an eigenstate of $\mathrm{Sz}, \mid \mathrm{z}+>$ with probability $|\alpha|^{2}$, and $\mid z->$ with probability $|\beta|^{2}$ ") or at the whole beam (e.g. "it is not in an eigenstate of $\mathrm{Sz}$ because it is a statistical mixture, with $\alpha$ and $\beta$ identifying the fraction of atoms with spin up and spin down"). In interviews, students clearly explained the classical roots of their reasoning: "They [the atoms] are in an eigenstate of Sz because the device didn't modify their spin. It deflected them by means of a magnetic field precisely on the base of their initial spin component Sz".

A notable aspect of this kind of reasoning is that it can be harmonized with the concept of collapse. A student stated in his written answer: "We discover the spin value along $\mathrm{z}$ axis and the system collapses into an eigenstate". Only in the interview it was possible to clear up the conceptual tension evident in his statement: "We have a mixture of small balls with spin up and down. By measuring, we isolate one of them".

Four undergraduates and one graduate stated that the system is already in an eigenstate of $\mathrm{Sz}$, giving alternative interpretations of the concept of eigenstate: "if on those atoms it is possible to measure $\mathrm{Sz}$, then they are in an eigenstate of Sz", or "yes. They are in an eigenstate which, summed up, gives us Sz". These students showed difficulties with the very concept of eigenstate.

Comparing answers to Q1 with answers to Q2, we see that all students but one discussing the concept of phase in Q1 correctly answered Q2. All students but one displaying a passive/classic concept of measurement in $\mathrm{Q} 2$ considered $\alpha=\beta \in R^{+}$in $\mathrm{Q} 1$.

Q3: By means of the same source described in Q1, a beam of silver atoms is prepared in the same state $|\psi\rangle$ as before. The Stern-Gerlach device is replaced by a similar one, measuring the spin component in the $\mathrm{x}$ direction $(\mathrm{Sx})$.

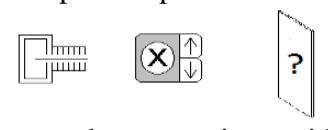

Is it possible that we observe again two identical spots on the screen?

To answer the question, it may be handy to use the following relations: $\quad \mid z+>=(|x+>+| x->) / \sqrt{2}$

$\mid z->=(|x+>-| x->) / \sqrt{2}$

FIGURE 3. Item Q3's text. 
Q3 resulted to be a difficult task for students. This was to be expected, as its resolution requires a consistent interpretation and use of the basis change equations, as well as of information gained in question Q1. 9/32 undergraduates didn't answer the item. Of course, as the answer given to Q1 influences reasoning in facing Q3, in this analysis we take into account the results of Q1.

Among the six students discussing phase relations in Q1, only half correctly answered Q3, identifying the significance of phase in the experimental prediction. They applied the change of basis and, assuming $\beta=$ $e^{i \varphi} 1 / \sqrt{2}$, they came to the conclusion. One of these students said in his answer to Q1 that "phase is irrelevant from a physical point of view". In the interview, he corrected his statement: "this is not always true. In time evolution, phase can give rise to cosine". Noteworthy, even after answering Q3, he associated phase only to time evolution. Only in later stages of the discussion on Q3, he observed that "phase corresponds to information on the system we didn't get in the first measurement". The other three students didn't use phase in Q3 and, consistently interpreting basis change equations, concluded that $|\psi\rangle=|x+\rangle$. This result is compatible with the idea that phase is physically unimportant (which is true for an overall factor, but not for phase difference).

Of the five students who correctly answered Q1 by writing " $|\alpha|^{2}=|\beta|^{2}=1 / 2$ ", only one undergraduate and one graduate took phase into account, while three other graduates concluded that $|\psi\rangle=|x+\rangle$.

Considering those students who in Q1 had stated that $\alpha=\beta$, half of them (9/21 undergraduates, $2 / 3$ graduates) consistently interpreted the change of basis formulas, either concluding that $|\psi\rangle=|x+\rangle$, or stating that $\alpha=\beta$ is a necessary condition to see again two identical spots.

Six undergraduates and one graduate didn't apply the change of basis, claiming that two equally bright spots would appear, and justifying this prediction with the claim that measurements on different axes are independent. "If the new device produces a magnetic field $\mathbf{B}=\mathrm{B} \hat{x}$, then I'll obtain two equally bright spots" or "if the magnetic field is oriented along $\mathrm{x}$ axis, we are in the same situation as before". This is consistent with previous findings [2]. Four of these students had answered Q2 by interpreting the beam as a statistical mixture of states. This could partly explain their conclusion on Q3: "The atoms are equally divided between $|z+\rangle$ e $\mid z->$. By measuring a different spin component, I should obtain the same effect."

\section{CONCLUSIONS}

In transposing patterns of experimental data into quantum state formalism, most students focused exclusively on square modulus, neglecting phase and considering coefficients as $\mathfrak{R}^{+}$numbers. This represents an obstacle in recognizing the distinction between measurement and state reconstruction (RQ1).

As a related issue, a significant fraction of students answering Q2 (13/32) either identified a superposition state with an eigenstate or interpreted measurement as a passive classical process. The latter are among those who considered coefficients as $\mathfrak{R}^{+}$numbers (RQ2).

Even 6/11 students correctly answering Q1 didn't use phase relations in Q3 to make predictions on measurements of an observable not commuting with that on which $|\psi\rangle$ is expanded. This is compatible with an interpretation of phase difference as a needed formal element, but without physical meaning, or as the overall phase factor. Most students applied the change of basis in their answers to Q3, but 7/31 didn't, claiming that a measurement of spin on an axis doesn't influence a measurement on another. Four of these answers are compatible with an interpretation of $|\psi\rangle$ as a statistical mixture (RQ3).

Tested graduate students showed significantly better performances than undergraduates in Q1 (4/8 vs. 7/32 correct answers), equal to undergraduates in Q2, and only slightly better in Q3 (1/8 vs. 3/32). The two groups of students gave about the same alternative answers. Within the limits of our sample, it is possible to say that difficulties with basic quantum concepts are not necessarily solved in more advanced courses.

A need emerges to explicitly address the translation processes between mathematics and physical meaning already in undergraduate courses, discussing the structural role of Hilbert space constructs in the theory, as concerns the way in which information is encoded in the formal representations of quantum state.

\section{ACKNOWLEDGEMENTS}

We are very grateful to Professor M. Anselmino, Professor A. Devoto, and Professor V. Lubicz for administering the test to their students.

\section{REFERENCES}

1. R. Karam, Phys. Rev. ST PER, 10, 010119 (2014).

2. C. Singh, Am. J. Phys., 76(3), 277 (2008).

3. S. Goldhaber, S. Pollock, M. Dubson, P. Beale, and K. K. Perkins, in 2009 PERC Proceedings, pp. 145-148.

4. A. Crouse, Ph.D. dissertation, University of Washington, Seattle, 2007.

5. G. Zhu and C. Singh, Am. J. Phys., 80 (3), 252 (2012).

6. R. Duit, H. Gropengießer, and U. Kattmann, in Developing standards in research on science education, edited by $\mathrm{H}$. E. Fischer (2005), pp. 1-9.

7. G. Zuccarini, M. Michelini, A. Stefanel, in Proceedings of ESERA 2013, Nicosia, Cyprus.

8. G. Zuccarini, Il Nuovo Cimento C, 37(4), 273 (2014).

9. C. Singh, Am. J. Phys., 69 (8), 885 (2001). 